\title{
Eventually Periodic Solutions of a Max-Type System of Difference Equations of Higher Order
}

\author{
Guangwang Su $(\mathbb{D}$, Taixiang Sun, and Bin Qin \\ College of Information and Statistics, Guangxi University of Finance and Economics, Nanning 530003, China \\ Correspondence should be addressed to Guangwang Su; slg6w3@163.com
}

Received 24 January 2018; Accepted 21 March 2018; Published 24 April 2018

Academic Editor: Douglas R. Anderson

Copyright (C) 2018 Guangwang Su et al. This is an open access article distributed under the Creative Commons Attribution License, which permits unrestricted use, distribution, and reproduction in any medium, provided the original work is properly cited.

We study in this paper the following max-type system of difference equations of higher order: $x_{n}=\max \left\{A, y_{n-k} / x_{n-1}\right\}$ and $y_{n}=$ $\max \left\{B, x_{n-k} / y_{n-1}\right\}, n \in\{0,1,2, \ldots\}$, where $A \geq B>0, k \geq 1$, and the initial conditions $x_{-k}, y_{-k}, x_{-k+1}, y_{-k+1}, \ldots, x_{-1}, y_{-1} \in(0,+\infty)$. We show that (1) if $A B>1$, then every solution of the above system is periodic with period 2 eventually. (2) If $A B=1>B$, then every solution of the above system is periodic with period $2 k$ or 2 eventually. (3) If $A=B=1$ or $A B<1$, then the above system has a solution which is not periodic eventually.

\section{Introduction}

Our purpose in this paper is to study eventual periodicity of the following max-type system of difference equations of higher order:

$$
\begin{aligned}
& x_{n}=\max \left\{A, \frac{y_{n-k}}{x_{n-1}}\right\}, \\
& y_{n}=\max \left\{B, \frac{x_{n-k}}{y_{n-1}}\right\}, \\
& n \in \mathbb{N}_{0} \equiv\{0,1, \ldots\}
\end{aligned}
$$

with

$$
A \geq B>0,
$$

where $k \in \mathbb{N} \equiv\{1,2, \ldots\}$ and the initial conditions $x_{-k}, y_{-k}$, $x_{-k+1}, y_{-k+1}, \ldots, x_{-1}, y_{-1} \in \mathbb{R}_{+} \equiv(0,+\infty)$.

Recently, there has been a great interest in studying maxtype systems of difference equations. For example, Fotiades and Papaschinopoulos [1] studied the following max-type system of difference equations:

$$
\begin{aligned}
& x_{n}=\max \left\{A, \frac{y_{n-1}}{x_{n-2}}\right\}, \\
& y_{n}=\max \left\{B, \frac{x_{n-1}}{y_{n-2}}\right\},
\end{aligned}
$$

and they showed that every positive solution of (3) is eventually periodic.

In 2012, Stević [2] obtained in an elegant way the general solution to the following max-type system of difference equations:

$$
\begin{aligned}
& x_{n+1}=\max \left\{\frac{A}{x_{n}}, \frac{y_{n}}{x_{n}}\right\}, \\
& y_{n+1}=\max \left\{\frac{A}{y_{n}}, \frac{x_{n}}{y_{n}}\right\},
\end{aligned}
$$

$$
n \in \mathbb{N}_{0},
$$

for the case $x_{0}, y_{0} \geq A>0$ and $y_{0} / x_{0} \geq \max \{A, 1 / A\}$.

In [3], we have recently studied the following max-type system of difference equations:

$$
\begin{aligned}
& x_{n}=\max \left\{\frac{1}{x_{n-m}}, \min \left\{1, \frac{A}{y_{n-r}}\right\}\right\}, \\
& y_{n}=\max \left\{\frac{1}{y_{n-m}}, \min \left\{1, \frac{B}{x_{n-t}}\right\}\right\},
\end{aligned}
$$

$$
n \in \mathbb{N}_{0},
$$

where $A, B \in \mathbb{R}_{+}, m, r, t \in \mathbb{N}$, and the initial conditions $x_{-d}$, $y_{-d}, x_{-d+1}, y_{-d+1}, \ldots, x_{-1}, y_{-1} \in \mathbb{R}_{+}$with $d=\max \{m, r, t\}$, 
and we showed that every positive solution of (5) is eventually periodic with period $2 m$.

When $m=r=t=1$ and $A=B$, (5) reduces to the maxtype system of difference equations

$$
\begin{aligned}
& x_{n}=\max \left\{\frac{1}{x_{n-1}}, \min \left\{1, \frac{A}{y_{n-1}}\right\}\right\}, \\
& y_{n}=\max \left\{\frac{1}{y_{n-1}}, \min \left\{1, \frac{A}{x_{n-1}}\right\}\right\}, \\
& \qquad n \in \mathbb{N}_{0} .
\end{aligned}
$$

Yazlik et al. [4] in 2015 obtained in an elegant way the general solution of (6).

In 2014, Stević et al. [5] investigated the behavior of positive solutions of the following max-type system of difference equations:

$$
\begin{aligned}
& x_{n}=\max \left\{B, \frac{y_{n-1}^{p}}{z_{n-2}^{p}}\right\}, \\
& y_{n}=\max \left\{B, \frac{z_{n-1}^{p}}{x_{n-2}^{p}}\right\}, \\
& z_{n}=\max \left\{B, \frac{x_{n-1}^{p}}{y_{n-2}^{p}}\right\},
\end{aligned}
$$

$$
n \in \mathbb{N}_{0}
$$

where $B, p \in \mathbb{R}_{+}$. It is proved that system (7) is permanent when $p \in(0,4)$.

For some other results on solutions of many max-type difference equations and systems, such as eventual periodicity, the boundedness character, and attractivity, see, for example, [6-20] and the related references therein.

\section{Main Results and Proofs}

In this section, we study the eventual periodicity of solutions of system (1). Let $\left\{\left(x_{n}, y_{n}\right)\right\}_{n \geq-k}$ be a solution of (1) with the initial conditions $x_{-k}, y_{-k}, x_{-k+1}, y_{-k+1}, \ldots, x_{-1}, y_{-1} \in \mathbb{R}_{+}$. From (1), it immediately follows that, for any $n \in \mathbb{N}_{0}$,

$$
\begin{aligned}
& x_{n} \geq A, \\
& y_{n} \geq B .
\end{aligned}
$$

Lemma 1. If $x_{n}=A$ eventually, then $y_{n}$ is a periodic sequence with period 2 eventually. If $y_{n}=B$ eventually, then $x_{n}$ is a periodic sequence with period 2 eventually.

Proof. Assume that $x_{n}=A$ eventually. By (1), we see

$$
y_{n}=\max \left\{B, \frac{A}{y_{n-1}}\right\} \quad \text { eventually, }
$$

which implies

$$
y_{n} y_{n-1} \geq A \quad \text { eventually. }
$$

We have from (9), (10), and (11) that

$$
B \leq y_{n}=\max \left\{B, \frac{A y_{n-2}}{y_{n-1} y_{n-2}}\right\} \leq \max \left\{B, y_{n-2}\right\}=y_{n-2}
$$

eventually.

Then, for any $i \in\{0.1\}, y_{2 n+i}$ is eventually nonincreasing.

We claim that, for every $i \in\{0.1\}, y_{2 n+i}$ is a constant sequence eventually. Assume on the contrary that, for some $i \in\{0.1\}, y_{2 n+i}$ is not a constant sequence eventually. Then, by (10) and (12), we see that there exists a sequence of positive integers $k_{1}<k_{2}<\cdots$ such that, for any $n \in \mathbb{N}$,

$$
B<y_{2 k_{n+1}+i}=\frac{A}{y_{2 k_{n+1}+i-1}}<y_{2 k_{n}+i}=\frac{A}{y_{2 k_{n}+i-1}},
$$

which implies $y_{2 k_{n+1}+i-1}>y_{2 k_{n}+i-1}$ for any $n \in \mathbb{N}$. This is a contradiction. Thus, $y_{n}$ is a periodic sequence with period 2 eventually. The other case is treated similarly/dually, so we omit the detail. The proof is complete.

Lemma 2. If $A B \geq 1$, then $x_{2(n+1) k+i} \leq x_{2 n k+i}$ for any $n \geq k+1$ and $i \in \mathbb{N}_{0}$.

Proof. Assume that $A B \geq 1$. Then, for $2(n+1) k+i \geq k+1$, by (1) and (8), we have

$$
\begin{aligned}
A & \leq x_{2(n+1) k+i} \\
& =\max \left\{A, \frac{B}{x_{2(n+1) k+i-1}}, \frac{x_{2 n k+i}}{x_{2(n+1) k+i-1} y_{2(n+1) k+i-k-1}}\right\}
\end{aligned}
$$

and by (2), (8), and (9), we have

$$
\begin{aligned}
\frac{B}{x_{2(n+1) k+i-1}} & \leq \frac{B}{A} \leq 1 \leq A, \\
x_{2(n+1) k+i-1} y_{2(n+1) k+i-k-1} \geq A B & \geq 1 .
\end{aligned}
$$

Thus,

$$
x_{2(n+1) k+i} \leq \max \left\{A, x_{2 n k+i}\right\}=x_{2 n k+i} \text { eventually. }
$$

The proof is complete.

Theorem 3. Let $A B>1$. Then, $x_{n}=A$ eventually and $y_{n}$ is a periodic sequence with period 2 eventually.

Proof. Assume that $A B>1$. For any $i \in\{0.1, \ldots, 2 k-1\}$ and $n \in \mathbb{N}_{0}$, let $\lim _{n \rightarrow \infty} x_{2 n k+i}=A_{i}$ since $x_{2 n k+i}$ is eventually nonincreasing. Thus, by using (15) and by letting $n \rightarrow \infty$ in (14), it is obtained that $x_{n}=A$ eventually. By Lemma 1 , we see that $y_{n}$ is a periodic sequence with period 2 eventually. The proof is complete.

Now, we assume $A B=1$, and by Lemma 2, we can assume that, for any $i \in \mathbb{N}_{0}$,

$$
\lim _{n \rightarrow \infty} x_{2 n k+i}=A_{i} .
$$

Then, $A_{i} \geq A$ and $A_{2 k+i}=A_{i}$ for any $i \in \mathbb{N}_{0}$. 
Lemma 4. If $A B=1$ and $A_{i}>A$ for some $i \in \mathbb{N}_{0}$, then, for any $s \in \mathbb{N}, x_{2 n k+s+i}$ and $y_{2 n k-k+s+i}$ are constant sequences eventually.

Proof. Assume that $A B=1$ and $A_{i}>A$ for some $i \in \mathbb{N}_{0}$. Since $A_{i}>A$, it follows from (14), (15), and Lemma 2 that

$$
\begin{aligned}
x_{2 n k+i} & =\max \left\{A, \frac{x_{2(n-1) k+i}}{x_{2 n k-1+i} y_{2 n k-k-1+i}}\right\} \\
& =\frac{x_{2(n-1) k+i}}{x_{2 n k-1+i} y_{2 n k-k-1+i}} \text { eventually. }
\end{aligned}
$$

By (9), (17), and (18), we have

$$
\begin{aligned}
B & \leq \lim _{n \rightarrow \infty} y_{2 n k-k-1+i}=\lim _{n \rightarrow \infty} \frac{x_{2(n-1) k+i}}{x_{2 n k+i} x_{2 n k-1+i}}=\frac{1}{A_{-1+i+2 k}} \\
& \leq \frac{1}{A}=B .
\end{aligned}
$$

This implies

$$
\begin{gathered}
\lim _{n \rightarrow \infty} x_{2 n k-1+i}=A, \\
\lim _{n \rightarrow \infty} y_{2 n k-k-1+i}=B .
\end{gathered}
$$

Note that $x_{2 n k+i}=\max \left\{A, y_{2 n k-k+i} / x_{2 n k-1+i}\right\}=y_{2 n k-k+i} /$ $x_{2 n k-1+i}>A$ eventually; it follows that

$$
\lim _{n \rightarrow \infty} y_{2 n k-k+i}=\lim _{n \rightarrow \infty} x_{2 n k+i} x_{2 n k-1+i}=A_{i} A .
$$

Since

$$
\begin{aligned}
x_{2 n k+1+i} & =\max \left\{A, \frac{x_{2(n-1) k+1+i}}{x_{2 n k+i} y_{2 n k-k+i}}\right\}, \\
\lim _{n \rightarrow \infty} \frac{x_{2(n-1) k+1+i}}{x_{2 n k+i} y_{2 n k-k+i}} & =\frac{A_{1+i}}{A_{i}^{2} A}<A_{1+i},
\end{aligned}
$$

we see that $x_{2 n k+1+i}=A$ eventually. This with (21) implies

$$
\lim _{n \rightarrow \infty} \frac{x_{2(n-1) k+1+i}}{y_{2 n k-k+i}}=\frac{A}{A A_{i}}<B
$$

From (23), it follows that

$$
y_{2 n k-k+1+i}=\max \left\{B, \frac{x_{2(n-1) k+1+i}}{y_{2 n k-k+i}}\right\}=B \quad \text { eventually. }
$$

By $x_{2(n-1) k+2+i} / y_{2 n k-k+1+i} \geq A / B \geq 1 \geq B$ eventually and (24), it follows that

$$
\begin{aligned}
y_{2 n k-k+2+i} & =\max \left\{B, \frac{x_{2(n-1) k+2+i}}{y_{2 n k-k+1+i}}\right\}=\frac{x_{2(n-1) k+2+i}}{B} \\
x_{2 n k+2+i} & =\max \left\{A, \frac{y_{2 n k-k+2+i}}{x_{2 n k+1+i}}\right\} \\
& =\max \left\{A, x_{2(n-1) k+2+i}\right\}=x_{2(n-1) k+2+i}
\end{aligned}
$$

eventually.

If $x_{2 n k+2+i}>A$ eventually, then, in a similar fashion, we can obtain the following:
(1) $x_{2 n k+3+i}=A$ eventually and $y_{2 n k-k+3+i}=B$ eventually.

(2) $x_{2 n k+4+i}$ and $y_{2 n k-k+4+i}$ are constant sequences eventually.

If $x_{2 n k+2+i}=A$ eventually, then $y_{2 n k-k+2+i}=A / B$ eventually and

$$
\begin{aligned}
y_{2 n k-k+3+i} & =\max \left\{B, \frac{x_{2(n-1) k+3+i}}{y_{2 n k-k+2+i}}\right\} \\
& =\max \left\{B, \frac{x_{2(n-1) k+3+i} B}{A}\right\}=\frac{x_{2(n-1) k+3+i} B}{A}
\end{aligned}
$$

eventually,

$$
\begin{aligned}
x_{2 n k+3+i} & =\max \left\{A, \frac{y_{2 n k-k+3+i}}{x_{2 n k+2+i}}\right\} \\
& =\max \left\{A, \frac{x_{2(n-1) k+3+i} B}{A^{2}}\right\} \quad \text { eventually. }
\end{aligned}
$$

From (27), we see that if $A=B=1$, then by $x_{2(n-1) k+3+i} \geq A$ we have

$$
x_{2 n k+3+i}=x_{2(n-1) k+3+i} \quad \text { eventually, }
$$

and if $A>B$, then

$$
x_{2 n k+3+i}=A \quad \text { eventually }
$$

since

$$
\lim _{n \rightarrow \infty} \frac{x_{2(n-1) k+3+i} B}{A^{2}}=\frac{A_{3+i} B}{A^{2}}<A_{3+i} .
$$

Using arguments similar to ones developed in the above given proof, we can show that, for any $s \in \mathbb{N}, x_{2 n k+s+i}$ and $y_{2 n k-k+s+i}$ are constant sequences eventually. The proof is complete.

Theorem 5. Assume $A B=1>B$. Then, one of the following statements holds:

(i) $x_{n}$ and $y_{n}$ are periodic sequences with period $2 k$ eventually.

(ii) $x_{n}=$ A eventually and $y_{n}$ is a periodic sequence with period 2 eventually.

Proof. Assume that $A=1 / B>B$. If $A_{i}>A$ for some $i \in \mathbb{N}_{0}$, then by Lemma 4 we see that, for any $s \in \mathbb{N}, x_{2 n k+s+i}$ and $y_{2 n k-k+s+i}$ are constant sequences eventually, which implies that $x_{n}$ and $y_{n}$ are periodic sequences with period $2 k$ eventually. In the following, we assume that $A_{i}=A$ for any $i \in \mathbb{N}_{0}$.

We claim that $x_{n}=A$ eventually. Indeed, if, for some $i \epsilon$ $\mathbb{N}_{0}, x_{2 n k+i}>A$, then by (1) we have

$$
x_{2 n k+i}=\max \left\{A, \frac{y_{2 n k-k+i}}{x_{2 n k-1+i}}\right\}=\frac{y_{2 n k-k+i}}{x_{2 n k-1+i}}
$$

eventually,

$$
x_{2 n k+1+i}=\max \left\{A, \frac{x_{2(n-1) k+1+i}}{x_{2 n k+i} y_{2 n k-k+i}}\right\} \text {, }
$$




$$
\begin{aligned}
\lim _{n \rightarrow \infty} \frac{x_{2(n-1) k+1+i}}{x_{2 n k+i} y_{2 n k-k+i}} & =\lim _{n \rightarrow \infty} \frac{x_{2(n-1) k+1+i}}{x_{2 n k+i}^{2} x_{2 n k-1+i}} \leq \frac{A_{1+i}}{A^{3}} \\
& <A_{1+i} .
\end{aligned}
$$

Then, we see that

$$
x_{2 n k+1+i}=A \text { eventually. }
$$

From (31), we have $y_{2 n k-k+i} \geq A^{2}$ eventually, and with (34) and $B=1 / A$, it follows that

$$
\begin{aligned}
& y_{2 n k-k+1+i}=\max \left\{B, \frac{x_{2(n-1) k+1+i}}{y_{2 n k-k+i}}\right\} \\
&=\max \left\{B, \frac{A}{y_{2 n k-k+i}}\right\}=B \quad \text { eventually, } \\
& y_{2 n k-k+2+i}=\max \left\{B, \frac{x_{2(n-1) k+2+i}}{y_{2 n k-k+1+i}}\right\}=\frac{x_{2(n-1) k+2+i}}{B} \\
& x_{2 n k+2+i}=\max \left\{A, \frac{y_{2 n k-k+2+i}}{x_{2 n k+1+i}}\right\} \\
&=\max \left\{A, x_{2(n-1) k+2+i}\right\}=x_{2(n-1) k+2+i} \\
& \text { eventually, } \\
& \text { eventually. }
\end{aligned}
$$

Thus, $x_{2 n k+2+i}$ and $y_{2 n k-k+2+i}$ are constant sequences eventually. Using arguments similar to ones developed in the proof of Lemma 4 , we can show that, for any $s \in \mathbb{N}, x_{2 n k+s+i}$ and $y_{2 n k-k+s+i}$ are constant sequences eventually. Thus, $x_{2 n k+i}=$ $A_{i}=A$ eventually; this is a contradiction.

By Lemma 1, we see that $x_{n}=A$ eventually and $y_{n}$ is a periodic sequence with period 2 eventually. Theorem 5 is proven.

Theorem 6. If $A \leq 1$ and $B \leq 1$, then (1) has a solution which is not periodic eventually.

Proof. We claim that the equation

$$
z_{n}=\frac{z_{n-k}}{z_{n-1}}
$$

has a nonincreasing solution. Indeed, write

$$
\begin{aligned}
& M=\left\{\left(x_{1}, \ldots, x_{k}\right): x_{1} \geq \cdots \geq x_{k} \geq \sqrt{x_{1}}>0\right\}, \\
& E=\left\{\left(x_{1}, \ldots, x_{k}\right): x_{1} \geq \cdots \geq x_{k} \geq \frac{x_{1}}{x_{k-1}}>0\right\} .
\end{aligned}
$$

It is easy to see that $M \subset E$ since, for any $\left(x_{1}, \ldots, x_{k}\right) \in M$, we have $x_{1} \geq \cdots \geq x_{k} \geq \sqrt{x_{1}}$ and $x_{k} \geq x_{k}^{2} / x_{k-1} \geq x_{1} / x_{k-1}$. Define $F: M \rightarrow E$ by

$$
F\left(x_{1}, \ldots, x_{k}\right)=\left(u_{1}, \ldots, u_{k}\right) \equiv\left(x_{2}, \ldots, x_{k}, \frac{x_{1}}{x_{k}}\right)
$$

for all $\left(x_{1}, \ldots, x_{k}\right) \in M$.

We claim that $F$ is well defined. Indeed, from (38) and the definition of $M$, we have

$$
u_{i}=x_{i+1}, \quad \text { for } i \in\{1, \ldots, k-1\},
$$

$$
\begin{aligned}
& u_{k}=\frac{x_{1}}{x_{k}} \\
& u_{1} \geq \cdots \geq u_{k-1}=x_{k} \geq \frac{x_{1}}{\sqrt{x_{1}}} \geq \frac{x_{1}}{x_{k}}=u_{k} \geq \frac{u_{1}}{u_{k-1}} .
\end{aligned}
$$

Thus, $\left(u_{1}, \ldots, u_{k}\right) \in E$.

We also claim that $F$ is a bijection from $M$ to $E$. Indeed, let $z=\left(z_{1}, \ldots, z_{k}\right), y=\left(y_{1}, \ldots, y_{k}\right) \in M$ with $z \neq y$; we have $F(y) \neq F(z)$. On the other hand, for any $u=\left(u_{1}, \ldots, u_{k}\right) \in E$, we have

$$
u_{1} \geq \cdots \geq u_{k} \geq \frac{u_{1}}{u_{k-1}}
$$

Choose

$$
x=\left(x_{1}, \ldots, x_{k}\right) \equiv\left(u_{k} u_{k-1}, u_{1}, \ldots, u_{k-1}\right) .
$$

It follows from (40) and (41) that

$$
\begin{aligned}
x_{1} & =u_{k} u_{k-1} \geq u_{1}=x_{2} \geq \cdots \geq u_{k-1}=x_{k}=\frac{u_{k-1} u_{k}}{u_{k}} \\
& \geq \frac{u_{k-1} u_{k}}{\sqrt{u_{k-1} u_{k}}}=\sqrt{x_{1}},
\end{aligned}
$$

which implies $x \in M$, and by (38) we obtain $F(x)=u$.

Furthermore, since $F^{-1}\left(u_{1}, \ldots, u_{k}\right)=\left(u_{k} u_{k-1}, u_{1}, \ldots\right.$, $\left.u_{k-1}\right)$ is continuous, $F$ is a homeomorphism from $M$ to E.

Since $M \subset E$ and $F$ is a homeomorphism from $M$ onto $E$, it follows that $F^{-1}(M) \subset F^{-1}(E)=M$. By induction, we have

$$
p=(1,1, \ldots, 1) \in F^{-n}(M) \subset F^{-n+1}(M)
$$

for every $n \in \mathbb{N}$. Because $M$ is an unbounded connected closed set, we know that $F^{-n}(M)$ is an unbounded connected closed set for every $n \in \mathbb{N}$. Let

$$
S=\bigcap_{i=0}^{\infty} F^{-i}(M)
$$

Then, $S \neq \emptyset$ and $S$ is also an unbounded connected set.

Now, let $\left\{z_{n}\right\}_{n=-k}^{\infty}$ be a solution of (36) with the initial conditions $\left(z_{-k}, \ldots, z_{-1}\right) \in S-\{p\}$; we can show that, for all $n \in \mathbb{N}$,

$$
F^{n}\left(z_{-k}, \ldots, z_{-1}\right)=\left(z_{n-k}, \ldots, z_{n-1}\right) \in M-\{p\} .
$$

Thus, $z_{n} \geq z_{n+1}>1$ for any $n \geq-k$ and $\lim _{n \rightarrow \infty} z_{n}=1$. It is easy to see that $\left\{\left(z_{n}, z_{n}\right)\right\}_{n=-k}^{\infty}$ is also a solution of (1) which is not periodic eventually. Theorem 6 is proven.

Theorem 7. If $A B<1<A$, then (1) has an unbounded solution.

Proof. Let $\left\{\left(x_{n}, y_{n}\right)\right\}_{n=-k}^{\infty}$ be a solution of (1) with the initial values $x_{i}=A$ and $y_{i}=B$ for $i \in\{-k,-k+1, \ldots,-1\}$. 
If $k=2 m$ for some $m \in \mathbb{N}$, then it is easy to see that, for $i \in$ $\{0,1, \ldots, m-1\}$, we have $\left(x_{2 i}, y_{2 i}\right)=(A, A / B),\left(x_{2 i+1}, y_{2 i+1}\right)=$ $(A, B)$. Thus,

$$
\begin{aligned}
& \left(x_{k}, y_{k}\right)=\left(\max \left\{A, \frac{y_{0}}{x_{k-1}}\right\}, \max \left\{B, \frac{x_{0}}{y_{k-1}}\right\}\right) \\
& =\left(\max \left\{A, \frac{1}{B}\right\}, \max \left\{B, \frac{A}{B}\right\}\right)=\left(\frac{1}{B}, \frac{A}{B}\right) \text {, } \\
& \left(x_{k+1}, y_{k+1}\right)=\left(\max \left\{A, \frac{y_{1}}{x_{k}}\right\}, \max \left\{B, \frac{x_{1}}{y_{k}}\right\}\right) \\
& =\left(\max \left\{A, B^{2}\right\}, \max \{B, B\}\right)=(A, B) \text {, } \\
& \left(x_{k+2}, y_{k+2}\right)=\left(\max \left\{A, \frac{y_{2}}{x_{k+1}}\right\}, \max \left\{B, \frac{x_{2}}{y_{k+1}}\right\}\right) \\
& =\left(\max \left\{A, \frac{1}{B}\right\}, \max \left\{B, \frac{A}{B}\right\}\right)=\left(\frac{1}{B}, \frac{A}{B}\right) \text {, } \\
& \left(x_{k+3}, y_{k+3}\right)=\left(\max \left\{A, \frac{y_{3}}{x_{k+3}}\right\}, \max \left\{B, \frac{x_{3}}{y_{k+3}}\right\}\right) \\
& =\left(\max \left\{A, B^{2}\right\}, \max \{B, B\}\right)=(A, B) \text {, } \\
& \left(x_{2 k-2}, y_{2 k-2}\right)=\left(\max \left\{A, \frac{y_{k-2}}{x_{2 k-3}}\right\}, \max \left\{B, \frac{x_{k-2}}{y_{k-3}}\right\}\right) \\
& =\left(\max \left\{A, \frac{1}{B}\right\}, \max \left\{B, \frac{A}{B}\right\}\right)=\left(\frac{1}{B}, \frac{A}{B}\right), \\
& \left(x_{2 k-1}, y_{2 k-1}\right) \\
& =\left(\max \left\{A, \frac{y_{k-1}}{x_{2 k-2}}\right\}, \max \left\{B, \frac{x_{k-1}}{y_{2 k-2}}\right\}\right) \\
& =\left(\max \left\{A, B^{2}\right\}, \max \{B, B\}\right)=(A, B), \\
& \left(x_{2 k}, y_{2 k}\right)=\left(\max \left\{A, \frac{y_{k}}{x_{2 k-1}}\right\}, \max \left\{B, \frac{x_{k}}{y_{2 k-1}}\right\}\right) \\
& =\left(\max \left\{A, \frac{1}{B}\right\}, \max \left\{B, \frac{1}{B^{2}}\right\}\right)=\left(\frac{1}{B}, \frac{1}{B^{2}}\right) \text {, } \\
& \left(x_{2 k+1}, y_{2 k+1}\right)=\left(\max \left\{A, \frac{y_{k+1}}{x_{2 k}}\right\}, \max \left\{B, \frac{x_{k+1}}{y_{2 k}}\right\}\right) \\
& =\left(\max \left\{A, B^{2}\right\}, \max \left\{B, B^{2} A\right\}\right)=(A, B), \\
& \left(x_{2 k+2}, y_{2 k+2}\right) \\
& =\left(\max \left\{A, \frac{y_{k+2}}{x_{2 k+1}}\right\}, \max \left\{B, \frac{x_{k+2}}{y_{2 k+1}}\right\}\right) \\
& =\left(\max \left\{A, \frac{1}{B}\right\}, \max \left\{B, \frac{1}{B^{2}}\right\}\right)=\left(\frac{1}{B}, \frac{1}{B^{2}}\right), \\
& \left(x_{2 k+3}, y_{2 k+3}\right) \\
& =\left(\max \left\{A, \frac{y_{k+3}}{x_{2 k+2}}\right\}, \max \left\{B, \frac{x_{k+3}}{y_{2 k+2}}\right\}\right) \\
& =\left(\max \left\{A, B^{2}\right\}, \max \left\{B, B^{2} A\right\}\right)=(A, B),
\end{aligned}
$$

$$
\begin{aligned}
& \left(x_{3 k-2}, y_{3 k-2}\right) \\
& =\left(\max \left\{A, \frac{y_{2 k-2}}{x_{3 k-3}}\right\}, \max \left\{B, \frac{x_{2 k-2}}{y_{3 k-3}}\right\}\right) \\
& =\left(\max \left\{A, \frac{1}{B}\right\}, \max \left\{B, \frac{1}{B^{2}}\right\}\right)=\left(\frac{1}{B}, \frac{1}{B^{2}}\right), \\
& \left(x_{3 k-1}, y_{3 k-1}\right) \\
& =\left(\max \left\{A, \frac{y_{2 k-1}}{x_{3 k-2}}\right\}, \max \left\{B, \frac{x_{2 k-1}}{y_{3 k-2}}\right\}\right) \\
& =\left(\max \left\{A, B^{2}\right\}, \max \left\{B, B^{2} A\right\}\right)=(A, B) .
\end{aligned}
$$

By induction, we can obtain that, for any $n \in \mathbb{N}$ and $i \in\{0,1$, $\ldots, m-1\}$,

$$
\begin{aligned}
\left(x_{(2 n-1) k+2 i}, y_{(2 n-1) k+2 i}\right) & =\left(\frac{1}{A^{n-1} B^{n}}, \frac{1}{A^{n-2} B^{n}}\right), \\
\left(x_{2 n k+2 i}, y_{2 n k+2 i}\right) & =\left(\frac{1}{A^{n-1} B^{n}}, \frac{1}{A^{n-1} B^{n+1}}\right), \\
\left(x_{n k+2 i+1}, y_{n k+2 i+1}\right) & =(A, B) .
\end{aligned}
$$

If $k=2 m+1$ for some $m \in \mathbb{N}$, then we also can obtain that, for any $n \in \mathbb{N}$,

$$
\begin{aligned}
&\left(x_{(2 n-1) k+2 i}, y_{(2 n-1) k+2 i}\right)=\left(\frac{1}{A^{n-1} B^{n}}, B\right) \\
&(i \in\{0,1, \ldots, m\}), \\
&\left(x_{(2 n-1) k+2 i+1}, y_{(2 n-1) k+2 i+1}\right)=\left(A, \frac{1}{A^{n-2} B^{n}}\right) \\
&(i \in\{0,1, \ldots, m-1\}), \\
&\left(x_{2 n k+2 i}, y_{2 n k+2 i}\right)=\left(A, \frac{1}{A^{n-1} B^{n+1}}\right) \\
&(i \in\{0,1, \ldots, m\}), \\
&\left(x_{2 n k+2 i+1}, y_{2 n k+2 i+1}\right)=\left(\frac{1}{A^{n-1} B^{n}}, B\right) \\
&(i \in\{0,1, \ldots, m-1\}) .
\end{aligned}
$$

It is easy to see that $\left\{\left(x_{n}, y_{n}\right)\right\}_{n=-k}^{\infty}$ is an unbounded solution of (1). Theorem 7 is proven.

\section{Conflicts of Interest}

The authors declare that there are no conflicts of interest regarding the publication of this paper.

\section{Acknowledgments}

This project was supported by the NNSF of China (Grant no. 11761011), NSF of Guangxi (Grants nos. 
2016GXNSFBA380235 and 2016GXNSFAA380286), and YMTBAPP of Guangxi Colleges (Grant no. 2017KY0598).

\section{References}

[1] N. Fotiades and G. Papaschinopoulos, "On a system of difference equations with maximum," Applied Mathematics and Computation, vol. 221, pp. 684-690, 2013.

[2] S. Stević, "Solutions of a max-type system of difference equations," Applied Mathematics and Computation, vol. 218, no. 19, pp. 9825-9830, 2012.

[3] T. Sun and H. Xi, "On the solutions of a system of difference equations with maximum," Applied Mathematics and Computation, vol. 290, pp. 292-297, 2016.

[4] Y. Yazlik, D. T. Tollu, and N. Taskara, "On the solutions of a maxtype difference equation system," Mathematical Methods in the Applied Sciences, vol. 38, no. 17, pp. 4388-4410, 2015.

[5] S. Stević, A. Alotaibi, N. Shahzad, and M. A. Alghamdi, "On positive solutions of a system of max-type difference equations," Journal of Computational Analysis and Applications, vol. 16, no. 5, pp. 906-915, 2014.

[6] K. S. Berenhaut, J. D. Foley, and S. Stević, "Boundedness character of positive solutions of a max difference equation," Journal of Difference Equations and Applications, vol. 12, no. 12, pp. 1193-1199, 2006.

[7] E. M. Elsayed, B. D. Iricanin, and S. Stević, "On the max-type equation $x_{n+1}=\max \left\{A_{n} / x_{n}, x_{n-1}\right\}$," Ars Combi, vol. 9, p. 187, 2010.

[8] W. Liu, X. Yang, and S. Stević, "On a class of nonautonomous max-type difference equations," Abstract and Applied Analysis, Art. ID 436852, 15 pages, 2011.

[9] B. Qin, T. Sun, and H. Xi, "Dynamics of the max-type difference equation $x_{n+1}=\max \left\{A / x_{n}, x_{n-k}\right\}$," Journal of Computational Analysis and Applications, vol. 14, no. 5, pp. 856-861, 2012.

[10] T. Sauer, "Global convergence of max-type equations," Journal of Difference Equations and Applications, vol. 17, no. 1, pp. 1-8, 2011.

[11] M. Sharif and Abdul Jawad, "Interacting generalized dark energy and reconstruction of scalar field models," Modern Physics Letters A, vol. 28, no. 38, Article ID 1350180, 15 pages, 2013.

[12] S. Stević, "On a generalized max-type difference equation from automatic control theory," Nonlinear Analysis. Theory, Methods \& Applications. An International Multidisciplinary Journal, vol. 72, no. 3-4, pp. 1841-1849, 2010.

[13] S. Stević, "Periodicity of max difference equations," Utilitas Mathematica, vol. 83, pp. 69-71, 2010.

[14] S. Stević, "Product-type system of difference equations of second-order solvable in closed form," Electronic Journal of Qualitative Theory of Differential Equations, pp. 1-16, 2015.

[15] S. Stević, "Representation of solutions of bilinear difference equations in terms of generalized fibonacci sequences," Electronic Journal of Qualitative Theory of Differential Equations, vol. 2014, pp. 1-15, 2014.

[16] S. Stević, M. A. Alghamdi, A. Alotaibi, and N. Shahzad, "Boundedness character of a max-type system of difference equations of second order," Electronic Journal of Qualitative Theory of Differential Equations, vol. 2014, no. 45, pp. 1-12, 2014.

[17] T. Sun, J. Liu, Q. He, X.-H. Liu, and C. Tao, "Eventually periodic solutions of a max-type difference equation," The Scientific World Journal, vol. 2014, Article ID 219437, 2014.
[18] T. Sun, B. Qin, H. Xi, and C. Han, "Global behavior of the maxtype difference equation $x_{n+1}=\max \left\{1 / x_{n}, A_{n} / x_{n-1}\right\}$," Abstract and Applied Analysis, vol. 2009, Article ID 152964, 2009.

[19] T. Sun, H. Xi, C. Han, and B. Qin, "Dynamics of the maxtype difference equation $x_{n+1}=\max \left\{1 / x_{n-m}, A_{n} / x_{n-r}\right\}$," Applied Mathematics and Computation, vol. 38, no. 1-2, pp. 173-180, 2012.

[20] Q. Xiao and Q.-h. Shi, "Eventually periodic solutions of a maxtype equation," Mathematical and Computer Modelling, vol. 57, no. 3-4, pp. 992-996, 2013. 


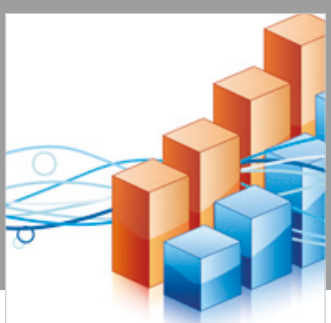

Advances in

Operations Research

\section{-n-m}
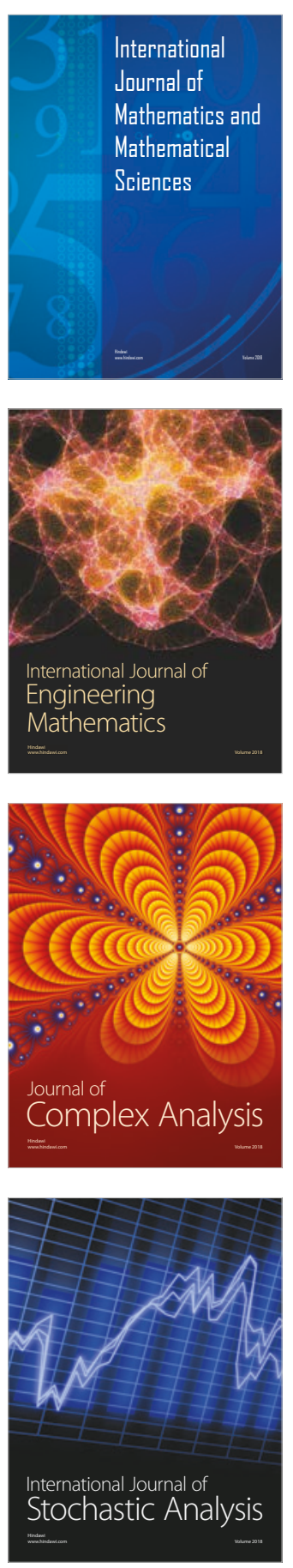
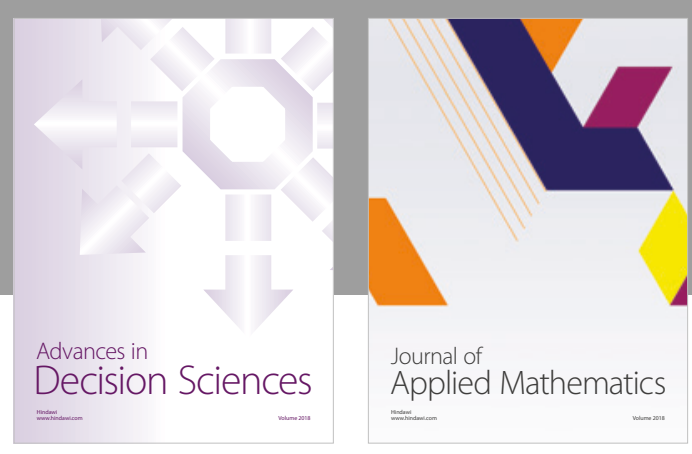

Journal of

Applied Mathematics
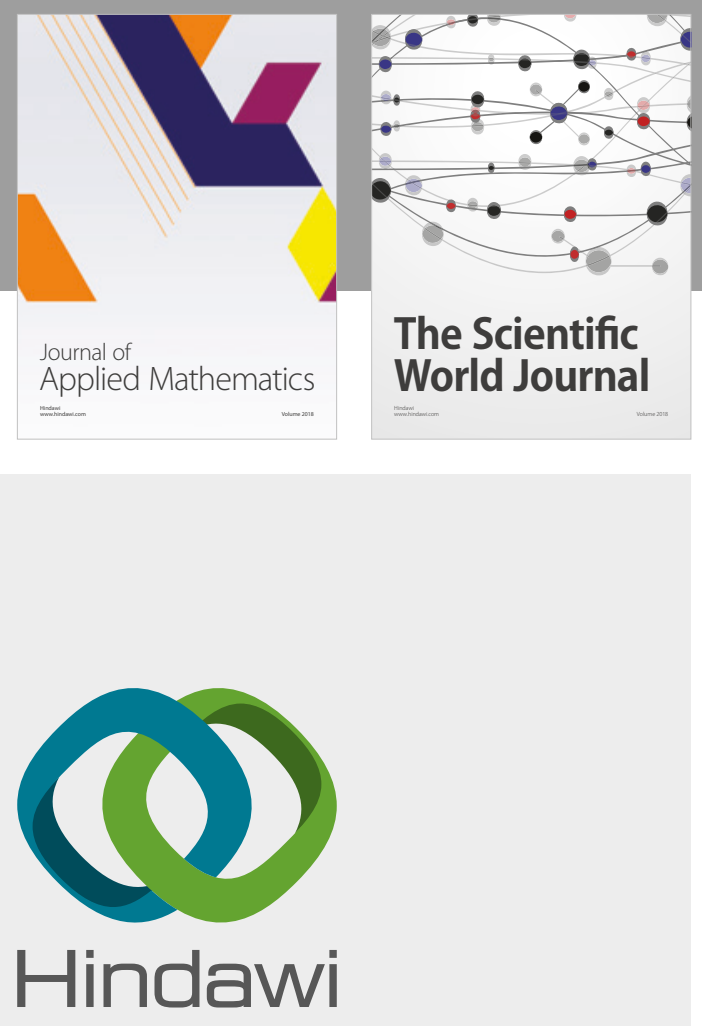

Submit your manuscripts at

www.hindawi.com

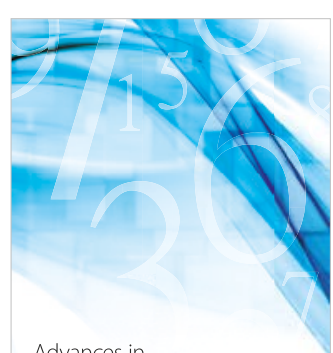

Advances in
Numerical Analysis
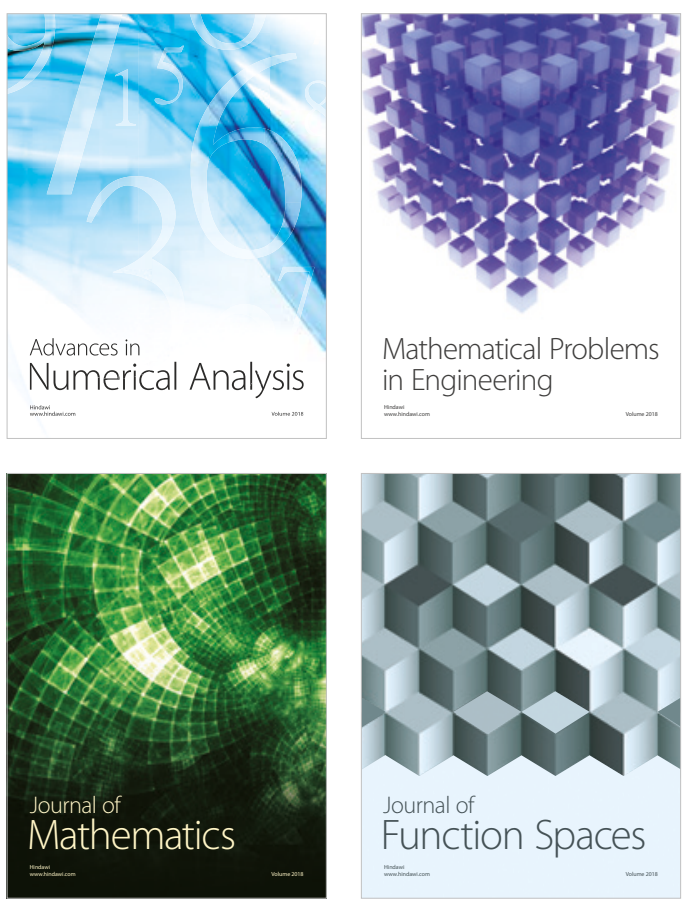

Mathematical Problems in Engineering

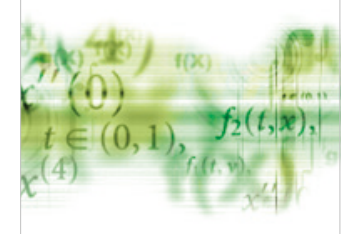

International Journal of

Differential Equations

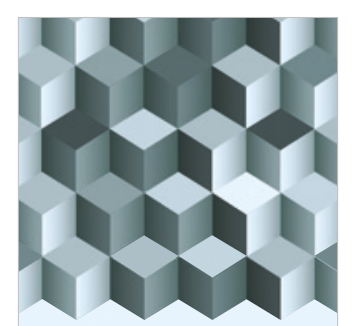

Journal of

Function Spaces

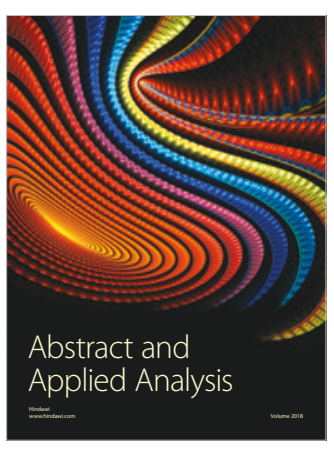

The Scientific

World Journal

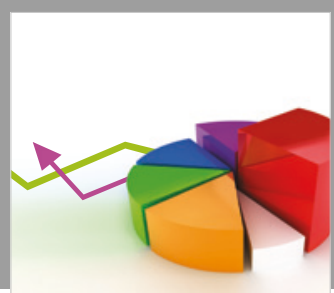

Journal of

Probability and Statistics
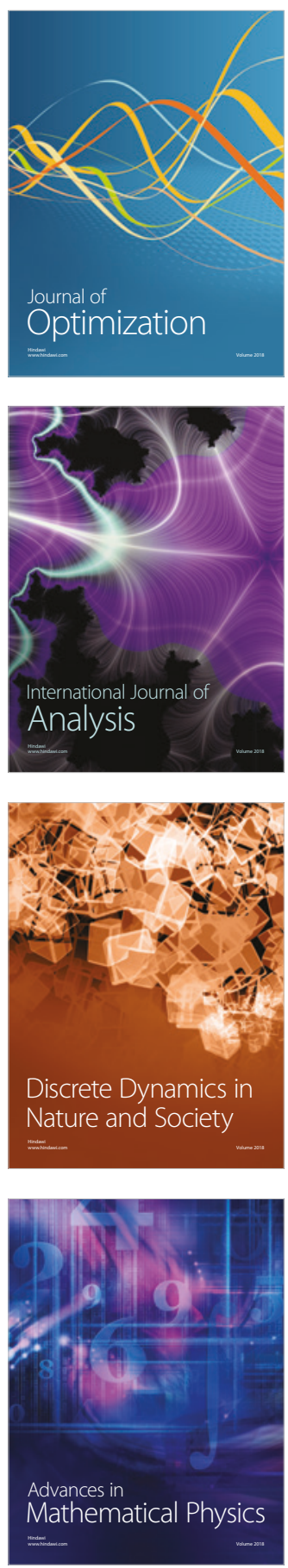